\title{
1 Widespread alterations in microRNA biogenesis in human Huntington's disease
}

\section{2 putamen}

4 Serena Petry ${ }^{1}$, Rémi Keraudren ${ }^{1}$, Behnaz Nateghi ${ }^{1}$, Andréanne Loiselle ${ }^{1}$, Karolina 5 Pircs $^{2,3,4}$, Johan Jakobsson ${ }^{4}$, Chantelle Sephton ${ }^{5,6}$, Mélanie Langlois ${ }^{7,8}$, Isabelle St6 Amour ${ }^{5,9, \#, \text { Sébastien S. Hébert }}{ }^{1,6, \#}$

$8{ }^{1}$ Centre de recherche du CHU de Québec-Université Laval, CHUL, Axe Neurosciences, 9 Québec, Canada.

102 HCEMM-SU, Neurobiology and Neurodegenerative Diseases Research Group,

11 Budapest, Hungary.

$12{ }^{3}$ Institute of Translational Medicine, Semmelweis University, Budapest, Hungary.

$13{ }^{4}$ Laboratory of Molecular Neurogenetics, Department of Experimental Medical Science, 14 Wallenberg Neuroscience Center and Lund Stem Cell Center, Lund University, Lund, 15 Sweden.

$16{ }^{5}$ Centre de recherche CERVO, Centre Intégré Universitaire de Santé et des Services

17 Sociaux de la Capitale-Nationale, Québec, Canada.

$18{ }^{6}$ Faculté de médecine, Département de psychiatrie et de neurosciences, Université Laval,

19 Québec, Canada

$20{ }^{7}$ Clinique des troubles du mouvement du CHU de Québec - Hôpital de l'Enfant-Jésus, Axe

21 Neurosciences, Québec, Canada.

$22{ }^{8}$ Faculté de médecine, Département des sciences neurologiques, Université Laval, Québec, 23 Canada. 
$24 \quad{ }^{9}$ Faculté de pharmacie, Université Laval, Québec, Canada.

25

26 \# Co-corresponding authors:

27 Dr. Isabelle St-Amour

28 Centre de recherche CERVO,

29 Centre Intégré Universitaire de Santé et des Services Sociaux de la Capitale-Nationale,

30 Québec, QC

31 G1E 1T2, Canada

32 Email: isabelle.st-amour@fmed.ulaval.ca

34 Dr. Sébastien S. Hébert

35 Centre de recherche du CHU de Québec-Université Laval, CHUL

362705 boul. Laurier

37 Neurosciences, P0-9800

38 Québec, QC

39 G1V 4G2, Canada

40 Email: sebastien.hebert@crchudequebec.ulaval.ca

42 Abstract

43 Altered microRNA (miRNA) expression is a common feature of Huntington's disease

44 (HD) and could participate in disease onset and progression. However, little is known about

45 the underlying causes of miRNA disruption in HD. We and others have previously shown

46 that mutant Huntingtin (mHTT) binds to Ago2, a central component of miRNA biogenesis, 
47 and disrupts mature miRNA levels. In this study, we sought to determine if miRNA

48 maturation per se was compromised in HD. Towards this end, we characterized t major

49 miRNA biogenesis pathway components and miRNA maturation products (pri-miRNA,

50 pre-miRNA, and mature) in human HD ( $\mathrm{N}=41$, Vonsattel grades HD2-4) and healthy

51 control $(\mathrm{N}=25)$ subjects. Notably, the striatum (putamen) and cortex (BA39) from the same

52 individuals were analyzed in parallel. We show that Ago2, Drosha, and Dicer were strongly

53 downregulated in human HD at early stages of disease. Using a panel of HD-related

54 miRNAs (miR-10b, miR-196b, miR-132, miR-212, miR-127, miR-128), we uncovered

55 various types of maturation defects in HD brain, the most prominent occurring at the pre-

56 miRNA to mature miRNA maturation step. Consistent with earlier findings, we provide

57 evidence that alterations in autophagy could participate in miRNA maturation defects.

58 Notably, most changes occurred in the striatum, which is more prone to HTT aggregation

59 and neurodegeneration. Likewise, we observed no significant alterations in miRNA

60 biogenesis in human HD cortex and blood, strengthening tissue-specific effects. Overall,

61 these data provide important clues into the underlying mechanisms behind miRNA

62 alterations in HD-susceptible tissues. Further investigations are now required to understand

63 the biological, diagnostic, and therapeutic implications of miRNA/RNAi biogenesis

64 defects in HD and related neurodegenerative disorders.

65

\section{Keywords}

67 Huntington's disease, microRNA, Ago2, Dicer, RNAi, Biogenesis, Autophagy

\section{Introduction}


70 Huntington's disease (HD) is an incurable, hereditary neurodegenerative disorder caused

71 by a CAG trinucleotide repeat expansion in exon 1 of the Huntingtin (Htt) gene. At the

72 protein level, this results in the generation of abnormal polyglutamine (PolyQ) repeats at

73 the N-terminus of Htt. HD typically manifests itself in midlife with motor and cognitive

74 symptoms associated with neurodegeneration in the striatum and to a lesser degree cortex.

75 The molecular mechanisms leading to Htt-mediated neurodegeneration are still unresolved,

76 although it is well recognized that abnormal regulation of gene expression is an early and

77 key feature of HD neuropathology [21, 30, 33].

79 The small non-coding microRNAs (miRNAs) play a central role in gene expression 80 regulation by promoting messenger RNA (mRNA) translation inhibition and/or 81 degradation $[41,42]$. MiRNA function is inherently related to its maturation that follows

82 two major processing steps $[12,27]$. First, the long primary miRNA transcript (pri-miRNA)

83 is cleaved by the Drosha/DGCR8 complex to generate a $\sim 70$ nucleotide (nt) precursor

84 miRNA (pre-miRNA). The pre-miRNA is transported to the cytoplasm by Exportin 5

85 where it is then cleaved by Dicer to generate a $\sim 22 \mathrm{nt}$ mature miRNA. The mature miRNA

86 is finally loaded with Ago2 and associated proteins (e.g., TRBP) into the endogenous

87 RNA-induced silencing complex (RISC) that binds to the 3'untranslated region (3'UTR)

88 of target mRNAs. Interestingly, miRNAs can control diverse biological pathways by

89 modulating one or several key target genes simultaneously [37]. Therefore, any disruption

90 in this pathway could have deleterious consequences on gene expression networks and cell

91 homeostasis. 
93 Indeed, it is now well established that miRNAs are essential to the survival of striatal and

94 cortical neurons $[5,13]$. Loss of neuronal Dicer in adult mice leads to alterations in

95 transcription, reduced brain size, behavioural defects, and decreased lifespan [6, 9],

96 reminiscent of some features of HD. In HD mice (YAC128 model), Lee et al. observed a

97 global upregulation or downregulation of mature miRNAs in early and late stages of

98 disease, respectively [23]. These changes coincided with transient changes in Dicer,

99 Drosha and Exportin mRNA levels. Recently, we and others have shown that mHtt binds

100 to Ago2 protein [29, 35, 36], whereas transient overexpression of mHtt in cells and mice

101 leads to higher Ago2 expression and widespread alterations in mature miRNA levels [29].

102 Furthermore, post-mortem studies have detected changes in mature miRNA expression

103 profiles in the brains of HD mice and humans [16-18, 22, 24, 25, 28].

104

105 Despite these observations, there is surprisingly no clear evidence that miRNA maturation

106 per se is defective in HD, especially in humans. This could have important implications in

107 understanding miRNA regulation and function within cell survival pathways and current

108 therapeutic efforts using the endogenous RISC (composed of Ago2 and Dicer) to silence

$109 \mathrm{mHtt}[1,8]$. Towards this end, we have analyzed, for the first time, all major miRNA

110 pathway components and maturation products (pri-miRNA, pre-miRNA, mature) in human

111 HD tissues samples. Notably, our experiments were conducted in different tissues that were

112 collected from patients at different stages of disease. In sum, our data implicate widespread

113 defects in the pre-miRNA to mature miRNA step in HD, which overlaps with mHtt

114 pathology and overt neurodegeneration in the striatum. 


\section{Materials and Methods}

\section{Human brain samples}

118 Dissected frozen human putamen and matching cortical (BA39 region) tissues (0,5-1,2 $\mathrm{g}$

119 per sample) were obtained from the Harvard Brain Tissues Resource Center via NIH

120 Neurobiobank (see Suppl. Table 1) as before [40]. This specific study included brain

121 tissues from 25 control and 41 HD individuals. Frozen post-mortem tissues were prepared

122 as described before and used for protein and RNA analysis [40]. CAG-repeat length was

123 determined by the CHU de Québec Sequencing and Genotyping platform using a 6-FAM

124 fluorescent primer (applied Biosystems Inc, Foster city, CA, USA) in a polymerase chain

125 reaction (PCR) followed by determination of the product size by capillary electrophoresis

126 in a 3130xl Genetic analyzers. We used the disease burden score (DBS) to estimate the

127 lifetime exposure to mutant huntingtin in individuals with HD with the following equation:

128 DBS = age at death $x($ CAG-repeat length - 35,5).

129

\section{Protein and RNA Extraction}

131 Total proteins were extracted as previously described [40]. In brief, frozen tissues were

132 mechanically homogenized in seven volumes of lysis buffer $(150 \mathrm{nM} \mathrm{NaCl}, 50 \mathrm{mM}$ Tris,

$1330.5 \%$ deoxycholate, $1 \%$ Triton $\mathrm{X}-100,0.5 \%$ sodium dodecyl sulfate (SDS), complete

134 protease inhibitor cocktail, $1 \mathrm{mM}$ of sodium fluoride and $1 \mathrm{mM}$ of activated orthovanadate 135 as phosphatase inhibitor), then sonicated three times for 5 X 1-s pulses. The solution was 136 spun at $100,000 \mathrm{~g}$ for $20 \mathrm{~min}$ at $4^{\circ} \mathrm{C}$. The supernatant (soluble proteins) was kept at $-80^{\circ} \mathrm{C}$ 137 until processed. The pellet was further homogenized in formic acid (FA) and spun for 20 $138 \min$ at $17,500 \mathrm{~g}$ at $4^{\circ} \mathrm{C}$. FA-soluble proteins (FA fraction) were dried before being sonicated 
139 in NuPAGE ${ }^{\circledR}$ LDS sample buffer (Life Technologies) supplemented with $0.1 \mathrm{M}$ of

140 dithiothreitol, incubated $10 \mathrm{~min}$ at $70^{\circ} \mathrm{C}$ and kept at $-80^{\circ} \mathrm{C}$ until processed. Soluble proteins

141 were quantified with Pierce ${ }^{\mathrm{TM}}$ BCA Protein Assay Kit (ThermoFisher Scientific) and

142 mixed to the NuPAGE ${ }^{\circledR}$ LDS sample buffer with 5\% final volume of $\beta$-mercaptoethanol,

143 then boiled $10 \mathrm{~min}$ at $95^{\circ} \mathrm{C}$ for Western blot analysis. Total RNA was extracted from frozen

144 tissues using TRIzol reagent (Ambion by Life Technologies) according to the

145 manufacturer's instructions. Total RNA pellet was suspended in RNase free water,

146 quantified, and diluted to a final concentration of $100 \mathrm{ng} / \mu \mathrm{L}$. RNA was kept at $-80^{\circ} \mathrm{C}$ until

147 processed for qRT-PCR analysis.

149 Western Blotting

150 Five to twenty micrograms of soluble proteins were separated by two different systems:

$151 \quad 10 \%$ SDS-polyacrylamide gels (SDS-Page) and gradient 3-15\% tris-acetate

152 polyacrylamide gels for higher and lower molecular weight proteins. For the 10\% SDS-

153 Page, proteins were transferred onto a $0.45 \mu \mathrm{m}$ nitrocellulose membrane (Bio-Rad, catalog

$\left.154 \mathrm{n}^{\circ} 1620115\right)$ for 1 hour at RT at 100V. For the gradient gels, proteins were transferred onto

155 a $0.45 \mu \mathrm{m}$ methanol-activated PVDF membrane (Immobilon, Millipore) overnight at $4{ }^{\circ} \mathrm{C}$

156 at $25 \mathrm{~V}$ and $45 \mathrm{~min}$ at $4^{\circ} \mathrm{C}$ at $75 \mathrm{~V}$ the next day. The membrane was blocked with $5 \%$ non-

157 fat milk and $1 \%$ bovine serum albumin, then incubated overnight at $4^{\circ} \mathrm{C}$ with the

158 appropriate primary antibodies (see Suppl. Table 3). On the second day, membranes were

159 incubated with appropriate secondary anti-IgG-HRP antibodies (Jackson

160 ImmunoResearch: anti-mouse, catalog $n^{\circ} 115-035-146$ or anti-rabbit, catalog $\mathrm{n}^{\circ} 111-035-$

161 144) at RT for $1 \mathrm{~h}$. The immune-reactive bands were acquired using Immobilon Western 
162 Chemiluminescent HRP Substrate (Millipore) and visualized with the Fusion FX (Vilber

163 Lourmat, Eberhardzell, Germany) imaging system. Normalization was performed on total

164 proteins obtained with Ponceau Red or StainFree staining. Band intensities were quantified

165 using the ImageJ software.

167 Dot blot

168 Two microliters of each sample were slowly spotted on nitrocellulose membrane. After

169 drying the membrane, non-specific sites were blocked, and membrane was processed as

170 described in the Western Blotting section. Dot intensity was normalized on the total amount

171 of tissue used for the extraction.

172

173 Primary microRNA Real Time Quantitative RT-PCR

174 The reverse transcription was performed with $500 \mathrm{ng}$ of total RNA using the High-capacity

175 cDNA reverse transcription kit (ThermoFisher Scientific, catalog $n^{\circ} 4368814$ ) according to

176 the manufacturer's instructions. Program: $25^{\circ} \mathrm{C}$ for $10 \mathrm{~min}, 37^{\circ} \mathrm{C}$ for $120 \mathrm{~min}$ and $85^{\circ} \mathrm{C}$ for

1775 min. cDNA was stored at $-20^{\circ} \mathrm{C}$ until further processing. The real-time quantitative PCR

178 (qRT-PCR) was performed with TaqMan Fast Advanced Master mix (ThermoFisher

179 Scientific, catalog $\left.\mathrm{n}^{\circ} 4444963\right)$ according to manufacturer’s instructions. Primers were

180 purchased from ThermoFisher Scientific (Hs03302879_pri, hsa-mir-10b;

181 Hs03303255_pri, hsa-mir-127; Hs03303101_pri, hsa-mir-128-1; Hs03303111_pri, hsa-

182 mir-132; Hs03293754_pri, hsa-mir-196b; Hs03302957_pri, hsa-mir-212). Primary

183 microRNAs were normalized to the geographic mean of GAPDH and RPL32. The relative 
184 amount of each primary microRNA was calculated using the comparative $\mathrm{Ct}\left(2^{-\Delta \Delta C t}\right)$

185 method as before [39].

187 Precursor microRNA Real Time Quantitative RT-PCR

188 The reverse transcription was performed with $500 \mathrm{ng}$ of total RNA using the miScript RT

189 II kit (Qiagen) according to the manufacturer's instructions. The RT-PCR was performed 190 with the Hiflex buffer, as recommended by the manufacturer to study precursor 191 microRNAs. Program: $38^{\circ} \mathrm{C}$ for $60 \mathrm{~min}$ and $95^{\circ} \mathrm{C}$ for $5 \mathrm{~min}$. cDNA was stored at $-20^{\circ} \mathrm{C}$ 192 until further processing. The qRT-PCR was performed with QuantiTect SYBR Green PCR 193 Master Mix (Qiagen) according to the manufacturer's instructions. miScript precursor 194 assay primers were purchased from Qiagen (mir-10b ID: MP00003983; mir-127-1 ID: 195 MP00004123; mir-128-1 ID: MP00004137; mir-132 MP00004179; mir-196b ID: 196 MP00004935; mir-212 ID: MP00004256). Precursor microRNAs were normalized to 197 SNORD95 (ID: MS00033726). The relative amount of each precursor microRNA was 198 calculated using the comparative $\mathrm{Ct}\left(2^{-\Delta \Delta C t}\right)$ method.

200 Mature microRNA Real Time Quantitative RT-PCR

201 The reverse transcription was performed with $10 \mathrm{ng}$ of total RNA using the TaqMan

202 MicroRNA Reverse transcription kit (ThermoFisher) according to the manufacturer's

203 instructions. Program: $16^{\circ} \mathrm{C}$ for $30 \mathrm{~min}, 42^{\circ} \mathrm{C}$ for $30 \mathrm{~min}$ and $85^{\circ} \mathrm{C}$ for $5 \mathrm{~min}$. cDNA was

204 stored at $-20^{\circ} \mathrm{C}$ until further processing. The qRT-PCR was performed with TaqMan Fast

205 Advanced Master mix (ThermoFisher Scientific) according to manufacturer's instructions.

206 miRNA assay primers were purchased from ThermoFisher Scientific (hsa-miR10b, 
207 002218; hsa-miR127, 000452; hsa-miR128, 002216; hsa-miR132, 000457; hsa-miR196b,

208 002215; hsa-miR212, 000515). Mature microRNAs were normalized to the geographic

209 mean of RNU48 (hsa-RNU48, 001006) and Let-7f (hsa-Let-7f, 000382). The relative

210 amount of each mature microRNA was calculated using the comparative $\mathrm{Ct}\left(2^{-\Delta \Delta C t}\right)$

211 method.

\section{Statistical Analysis}

214 All graphics and statistical analyses were performed using GraphPad Prism 9 Software

215 (Graph Pad Software, Inc., La Jolla, CA, USA). Normality and lognormality tests were

216 performed and parametric or non-parametric tests were used accordingly. When samples

217 distribution passed the normality test, a parametric one-way analysis of variance (ANOVA)

218 test followed by Dunnett's multiple comparisons and parametric unpaired student t-test

219 were performed. When samples distribution did not pass normality test, a non-parametric

220 Kruskal-Wallis test followed by Dunn's multiple comparisons and a non-parametric Mann

221 Whitney student t-test were performed. The threshold for statistical significance was set to

$222 p$-values $<0.05$.

\section{Results}

\section{Comparative biochemical analysis of Htt pathology in cortex and striatum}

226 In an attempt to understand the impact of endogenous human Htt on miRNA maturation,

227 we first evaluated Htt expression and pathology in two different brains regions affected in

228 human HD (see Suppl. Table 1). We quantified the amount of Htt protein in 41 HD patients

$229(\mathrm{~N}=10 \mathrm{HD} 2, \mathrm{~N}=23 \mathrm{HD} 3, \mathrm{~N}=8 \mathrm{HD} 4)$ and 25 Controls from matching striatal and cortical 
230 tissues. Consistent with earlier findings, we observed a decrease in soluble total (full-

231 length) Htt in HD striatum using 1HU-4C8 and CH00146 antibodies (Fig. 1A-E).

232 However, no significant changes in total Htt were shown in HD cortex. Using the 1HU-

$2334 \mathrm{C} 8$ clone, we detected an increase in N-terminal fragments ( $\mathrm{MW} \sim 40-50 \mathrm{KDa})$ in both

234 brain regions. As expected, an increase in formic acid (FA)-insoluble aggregated mHtt was

235 also observed in both regions using an anti-PolyQ antibody, although higher levels were

236 apparent in the striatum (Fig. 1H-I). Along with these results, significant decreases in

237 NeuN (neuronal marker), DARPP-32 (striatal neuron marker), and PSD-95 (post-synaptic

238 marker) protein levels were observed in HD striatum while the cortex was mostly spared

239 (Fig. 1L-N). Our results support that the relative expression level of these proteins is

240 modulated between brain regions (Suppl. Fig 1). These data suggest that Htt aggregation

241 is not the consequence of an increase expression level of endogenous Htt. Thus overall, in

242 line with previous results suggesting that HD pathology starts in the striatum, the striatal

243 tissue samples analyzed herein presented severe signs of Htt pathology and

244 neurodegeneration when compared to the cortex of the same individuals.

246 Early-stage alterations of miRNA pathway components in human HD striatum

247 Previous studies in mice $[23,29]$ showed that specific members of the miRNA biogenesis

248 pathway are compromised in HD models. In human brains, we observed a robust decrease

249 in Drosha, Dicer, and Ago2 protein levels from HD2 in the striatum but not in the cortex

250 (Fig. 2A-H). No significant changes in Dicer mRNA were noted in either region (Suppl.

251 Fig. 2), suggesting that alterations in expression occurred at the post-transcriptional level.

252 Modest or transient variations in DGCR8 and TRBP proteins were seen in these samples 
253 with no changes in Exportin 5. Taken together, these results suggest that core miRNA

254 biogenesis pathway components are rapidly and specifically compromised in human HD

255 striatum and precede overt neurodegeneration.

256

257 miRNA expression analysis in HD brain

258 Having shown that several major miRNA biogenesis components were compromised in 259 human HD brain, we next aimed to determine potential effects on miRNA levels. We 260 performed a literature search to identify HD-related miRNAs for downstream functional

261 analyses. Following an initial screen of 16 candidates previously associated with HD, we

262 selected a panel six conserved miRNAs that were commonly misregulated in both HD

263 striatum and cortex (Fig. 3 and Suppl. Fig. 3). These included miR-10b, miR-196b, miR-

264 127, miR-128, miR-132 and miR-212. To avoid any bias, we chose miRNAs that were

265 both upregulated and downregulated in HD. Our final choice was further influenced by the

266 different genomic sources of miRNAs: miR-10b and miR-196b are generated from introns

267 of host coding genes, miR-132 and miR-212 are co-expressed as a cluster from the same

268 non-coding gene, miR-127 is expressed from a much larger non-coding miRNA cluster,

269 whereas miR-128 is transcribed from an individual intergenic non-coding gene.

271 We quantified all three types of miRNA maturation products (primary, precursor, mature)

272 in the human striatum and cortex using a distinct set of normalization genes (Suppl. Fig.4).

273 As expected, we observed a co-expression of intronic miRNAs and host genes in HD (i.e.,

274 miR-10b and miR-19b in the striatum), as documented before (Fig. 3 and Suppl. Fig. 2).

275 Surprisingly, however, various other types of phenomena were observed outside from this 
276 canonical pattern, some of which were tissue and disease-stage specific. One example

277 includes the downregulation of pri-miR-127 and mature miR-127, but not pre-miR-127, in

278 HD striatum. Another example includes the specific downregulation of mature miR-132 in

279 HD striatum but an overall downregulation of pri-miR-132, pre-miR-132 and mature miR-

280132 in HD cortex. In sum, these results suggest that miRNA maturation is controlled at

281 both transcriptional and post-transcriptional levels in HD brain.

282

\section{Prominent pre-miRNA to mature miRNA maturation deficits in HD}

284 To better grasp any changes in miRNA maturation per se in HD, we analyzed overall ratios

285 (inhibition scores) between a given miRNA precursor and its substrate, as initially

286 proposed by Emde et al. [10]. The inhibition scores between pri-miRNA and pre-mRNA

287 were largely normal in HD striatum and cortex despite rare exceptions (Fig. 4A-F). On the

288 other hand, the inhibition scores between pre-mRNA and mature miRNA were drastically

289 altered for all tested miRNAs in HD striatum (Fig. 4G-L) whereas only miR-10b and miR-

$290196 \mathrm{~b}$ reached significance in late-stage HD cortex. Interestingly, miRNA levels and

291 inhibition scores were unaffected in human HD blood in a separate cohort (Suppl. Fig 5).

292 Taken together, these results implicate early and robust deficits in pre-miRNA to mature 293 miRNA maturation step in human HD striatum.

294

295 Autophagy dysfunction overlaps with miRNA maturation defects in HD

296 Finally, we set out to better understand the molecular mechanisms responsible for miRNA

297 maturation defects in HD. Autophagy dysfunction in an inherent feature of HD and we

298 have previously shown that it influences mature miRNA levels in vivo [29]. Accordingly, 
299 we observed a strong and significant downregulation of major markers of autophagy,

300 namely P62, LC3 and Beclin, in human HD striatum but not cortex at all stages of the

301 disease (Fig. 5A-G). Interestingly, TDP-43 levels, previously implicated in the regulation

302 of miRNA maturation in vitro [3, 19], did not correlate with miRNA defects $(\mathbf{5 A}, \mathbf{B}, \mathbf{H})$.

303 Taken together, these results strengthen a role for autophagy in modulating miRNA

304 maturation in HD-susceptible brain regions.

305

\section{Discussion}

307 This study provides the first in vivo evidence that miRNA maturation is dysregulated in

308 human HD and sheds a new light on the causes and potential implications of miRNA

309 dysregulation in HD. The importance of our findings is severalfold: 1) they provide

310 important clues on intra-individual variability and susceptibility towards $\mathrm{mHtt}$ pathology,

3112 2) they could explain a substantial proportion of miRNA alterations previously documented

312 in HD brain, 3) they provide a first in-depth analysis of the RISC components necessary

313 for endogenous RNA interference (RNAi), 4) they support the potential importance of

314 specific miRNAs (and downstream targets) in HD pathogenesis, and finally 5) they

315 strengthen the broad implications of autophagy dysregulation in HD pathogenesis.

317 To our knowledge, this is the first characterisation of human Htt expression and 318 aggregation in two different brain regions affected in HD from the same individuals. These 319 experiments validate and extend our previous biochemical studies focused on $\mathrm{Htt}$ 320 pathology and other proteinopathies exclusively in the striatum (putamen) [40]. In

321 agreement with earlier reports, lower $\mathrm{Htt}(\mathrm{mHtt})$ expression (loss-of-function) and higher 
$322 \mathrm{mHtt}$ aggregation (gain-of-function) are likely both contributing factors to the severe

323 neurodegeneration observed in the striatum. It is interesting to note that (at least some of)

324 the proposed toxic Htt N-terminal fragments [31, 44] were upregulated in both brain

325 regions analyzed, suggesting that additional or complementary factors participate in Htt-

326 mediated toxicity. This hypothesis is consistent with a role for miRNA-dependent survival

327 pathways in this process.

329 Remarkably, very little is known about the underlying causes of miRNA alterations in HD,

330 which is key to understanding the role, impact, diagnostic, and therapeutic potential of

331 miRNAs in human brain diseases. Clearly, the inhibitory effects of mHtt on transcription

$332[21,30,33]$ are readily evident in this study on both coding (e.g., miR-10b) and non-coding

333 (e.g., miR-127) genes their host miRNA transcripts. In addition to transcriptional effects,

334 our observations implicate other molecular mechanisms as major causes of mature miRNA

335 disruption in HD. The identification of factors that control pre-miRNA to miRNA

336 maturation abnormalities in HD (e.g., transport, cleavage, sequestration, degradation) will

337 require further investigation. Interestingly, cellular stress has been shown to disrupt pre-

338 miRNA to mature miRNA genesis in ALS [10]. Stress can influence miRNA maturation

339 in several ways, including the sequestration of pre-miRNAs and pathway components (e.g.,

340 Ago2) to $\mathrm{P}$ bodies and/or stress granules. On this line of thought, autophagy is functionally

341 implicated in $\mathrm{mHtt}$ protein turnover and aggregation, and more recently miRNA maturation

$342[2,43]$. Without a doubt, more studies are required to understand the cause-and-effect

343 relationship between these factors during HD progression. 
345 Interestingly, most miRNA biogenesis components were downregulated in human HD

346 striatum. This observation is somewhat consistent with earlier findings in mice that showed

347 a transient shift (up to down) in miRNA expression levels during disease progression. It

348 remains to be elucidated whether the triggering factor is a unique component (e.g., Ago2

349 downregulation [28]) or a more general mechanism in humans. The study of pre-

350 symptomatic HD patients (i.e., Vonsattel grades HD0-1) or humanized cell models (e.g.,

351 iPSC) would undoubtedly help to address this question. In any case, our results are

352 consistent with an abnormal regulation of miRNA biogenesis in HD.

353

354 Of note, we did not observe changes in mature miRNA levels (not shown) or miRNA

355 maturation defects in HD blood, although we and others have previously reported high

356 expression levels of Htt in blood cells [7, 32], further strengthening the hypothesis of

357 tissue-specific effects. At this stage, however, we cannot exclude maturation defects for

358 other miRNAs and/or cohorts. A critical question is how do mature miRNAs become

359 misregulated in tissues or cell types with seemingly normal miRNA biogenesis. As shown

360 herein, changes in gene transcription can lead to alternations of miRNA host genes and

361 henceforth mature miRNA output. In addition, and as mentioned above, mature miRNA

362 levels are subjected to multiple regulatory mechanisms (e.g., degradation) and feedback

363 loops that can go awry in disease conditions as well. An interesting hypothesis is that the

364 specific disruption of miRNA biogenesis - and not indirect effects of neurodegeneration

365 on mature miRNA levels - is responsible for the early susceptibility of cell loss in HD.

366 This could have context-specific consequences on key miRNAs or other RISC-dependent

367 RNAs that are required to maintain cell homeostasis. 
369 On this line of thought, several groups have already tested the regulatory effects of

370 candidate miRNAs on HD pathology, behavior, and cell survival. For example, an

371 increased expression of miR-196a (homologue of 196b) in transgenic mice caused lower

$372 \mathrm{mHtt}$ expression and aggregation in an HD model [4]. Overexpression of miR-10b in PC12

373 cells expressing mHtt also increased cell survival [16]. Finally, the brain supplementation

374 of miR-132 in HD mice partially rescued behavioral and motor symptoms [11].

375 Interestingly, the miR-132/212 cluster is among the most strongly affected miRNA

376 (family) in HD brain (this study and [11, 22]). We have previously shown that miR-

377 132/212 knockout mice display autophagy abnormalities and lower BDNF levels in the

378 brain as seen in $\operatorname{HD}[14,15,34,45]$. Additional studies are also required to establish the

379 underlying causes of Drosha, Dicer and Ago2 downregulation in HD striatum, although

380 autophagy is a reasonable candidate as well. The challenge now is to identify the targets

381 and pathways regulated by mature and possibly immature miRNA transcripts for in-depth

382 functional analyses in vivo, of course taking into account the occurrence of potential

383 transient changes as observed in HD mice and tissue-specific effects.

385 Interestingly, recent evidence suggests that impaired miRNA maturation occurs in other

386 trinucleotide repeat disorders as well. For instance, the expanded CGG repeats in FMRP

387 (causing Fragile X-associated tremor/ataxia syndrome) sequester DGCR8 and Drosha and

388 disrupt miRNA maturation in mice [38]. In drosophila, mutant ataxin-2 (causing

389 spinocerebellar ataxia type 2) disrupts Ago expression and miRNA function [26]. MiRNA

390 maturation is impaired also in models of expanded polyQ within ataxin-3 (causing 
391 Machado-Joseph disease), whereas blocking miRNA biogenesis increased ataxin-3

392 aggregation [20]. These observations strongly suggest that miRNA alterations in these

393 disorders are a direct consequence of disease genes (e.g., sequestration) rather than an

394 indirect effect of cell stress or other factors. The fact that Htt binds to Ago 2 is consistent

395 with this hypothesis although a role for additional genetic or molecular mechanisms cannot

396 be excluded in these diseases.

398 The endogenous RISC complex is central to the silencing of genes by miRNAs and other

399 small interfering RNAs such as small interfering RNAs (siRNAs). Interestingly, a variety

400 of therapeutic tools under development make use of miRNAs, siRNAs, or other antisense

401 oligonucleotides (including miRNA-like backbones) that silence mHtt expression in vivo

$402[1,8]$. As shown herein, the clinical testing of these compounds in human brain will need

403 to be carefully monitored for potential loss of RISC biological function. Clearly, much

404 more work is required to better understand the role and impact of miRNA biogenesis

405 abnormalities in HD and related trinucleotide disorders.

406

\section{Conclusions}

408 In summary, we show that pre-miRNA to mature miRNA biogenesis is strongly

409 compromised in human HD striatum. This observation could help to understand the

410 pathological relationship between Htt-Ago2 binding in vivo. Furthermore, this study

411 suggests that indirect or small changes in mature miRNA levels are not sufficient to

412 promote cell degeneration per se in trinucleotide diseases, compared to a "multiple-hit"

413 scenario implicating deficits in miRNA biogenesis or other RISC-dependent mechanisms. 
414 Finally, the results provided herein will guide current and future therapeutic strategies

415 involving the endogenous RISC in the human brain.

\section{List of abbreviations}

418 DiGeorge syndrome critical region 8, DGCR8; Human immunodeficiency virus

419 transactivating response RNA-binding protein; TRBP, Argonaut 2, Ago2; RNA-induced

420 silencing complex, RISC; Neuronal nuclear protein, NeuN; Dopamine- and cAMP-

421 regulated neuronal phosphoprotein, DARPP-32; Postsynaptic density protein 95, PSD95;

422 Brain-derived neurotrophic factor BDNF; nucleotide; nt.

423

\section{Acknowledgement}

425 This work was supported by the Canadian Institute of Health Research (CIHR, grant \# 426 272311), the Fonds de Recherche du Québec en Santé (FRQS), and the Huntington's

427 Disease Society of America (HDSA). The Harvard Brain Tissue Resource Center provided

428 tissues and is supported in part by HHSN-271-2013-00030C. The authors would like to

429 express a special appreciation to the nurses and staff who assisted in the collection and

430 storage of the human specimens. Finally, the authors are very grateful to all study 431 participants and their families who have contributed to this study.

\section{Ethics approval and consent to participate}

434 The experiments with human samples were approved by the CHU de Québec local 435 Research Ethics Committee (\#2017-3017). All work with human subjects was approved by 
436 the CHU de Québec human ethics committee (\#2020-4622) and in accordance with the

437 Declaration of Helsinki. Informed written consent was obtained from all participants.

438

\section{Competing interests}

440 The authors declare that they have no competing interests.

\section{References}

4431 Aguiar S, van der Gaag B, Cortese FAB (2017) RNAi mechanisms in Huntington's

444 disease therapy: siRNA versus shRNA. Transl Neurodegener 6: 30 Doi

$445 \quad 10.1186 / \mathrm{s} 40035-017-0101-9$

4462 Akkoc Y, Gozuacik D (2020) MicroRNAs as major regulators of the autophagy

447 pathway. Biochim Biophys Acta Mol Cell Res 1867: 118662 Doi

$448 \quad 10.1016 /$ j.bbamcr.2020.118662

4493 Buratti E, De Conti L, Stuani C, Romano M, Baralle M, Baralle F (2010) Nuclear

450 factor TDP-43 can affect selected microRNA levels. The FEBS journal 277: 2268-

$451 \quad 2281$ Doi 10.1111/j.1742-4658.2010.07643.x

4524 Cheng PH, Li CL, Chang YF, Tsai SJ, Lai YY, Chan AW, Chen CM, Yang SH

453 (2013) miR-196a ameliorates phenotypes of Huntington disease in cell, transgenic

454 mouse, and induced pluripotent stem cell models. American journal of human

455 genetics 93: 306-312 Doi 10.1016/j.ajhg.2013.05.025

4565 Chmielarz P, Konovalova J, Najam SS, Alter H, Piepponen TP, Erfle H, Sonntag

457 KC, Schutz G, Vinnikov IA, Domanskyi A (2017) Dicer and microRNAs protect

458 adult dopamine neurons. Cell Death Dis 8: e2813 Doi 10.1038/cddis.2017.214 
4596 Cuellar TL, Davis TH, Nelson PT, Loeb GB, Harfe BD, Ullian E, McManus MT 460 (2008) Dicer loss in striatal neurons produces behavioral and neuroanatomical 461 phenotypes in the absence of neurodegeneration. Proceedings of the National 462 Academy of Sciences of the United States of America 105: 5614-5619 Doi $463 \quad 10.1073 /$ pnas.0801689105

4647 Denis HL, Lamontagne-Proulx J, St-Amour I, Mason SL, Rowley JW, Cloutier N, 465 Tremblay ME, Vincent AT, Gould PV, Chouinard Set al (2019) Platelet 466 abnormalities in Huntington's disease. Journal of neurology, neurosurgery, and 467 psychiatry 90: 272-283 Doi 10.1136/jnnp-2018-318854

4688 Dong X, Cong S (2021) MicroRNAs in Huntington's Disease: Diagnostic 469 Biomarkers or Therapeutic Agents? Frontiers in cellular neuroscience 15: 705348 $470 \quad$ Doi $10.3389 /$ fncel.2021.705348

4719 Dorval V, Smith PY, Delay C, Calvo E, Planel E, Zommer N, Buee L, Hebert SS 472 (2012) Gene network and pathway analysis of mice with conditional ablation of 473 Dicer in post-mitotic neurons. PloS one 7: e44060 Doi $474 \quad$ 10.1371/journal.pone.0044060

47510 Emde A, Eitan C, Liou LL, Libby RT, Rivkin N, Magen I, Reichenstein I, 476 Oppenheim H, Eilam R, Silvestroni Aet al (2015) Dysregulated miRNA biogenesis 477 downstream of cellular stress and ALS-causing mutations: a new mechanism for 478 ALS. EMBO J 34: 2633-2651 Doi 10.15252/embj.201490493

47911 Fukuoka M, Takahashi M, Fujita H, Chiyo T, Popiel HA, Watanabe S, Furuya H, 480 Murata M, Wada K, Okada Tet al (2018) Supplemental Treatment for Huntington's 
Disease with miR-132 that Is Deficient in Huntington's Disease Brain. Molecular therapy Nucleic acids 11: 79-90 Doi 10.1016/j.omtn.2018.01.007

48312 Ha M, Kim VN (2014) Regulation of microRNA biogenesis. Nature reviews $484 \quad$ Molecular cell biology 15: 509-524 Doi 10.1038/nrm3838

48513 Hebert SS, Papadopoulou AS, Smith P, Galas MC, Planel E, Silahtaroglu AN, 486 Sergeant N, Buee L, De Strooper B (2010) Genetic ablation of Dicer in adult 487 forebrain neurons results in abnormal tau hyperphosphorylation and 488 neurodegeneration. Human molecular genetics 19: 3959-3969 Doi $10.1093 / \mathrm{hmg} / \mathrm{ddq} 311$

49014 Hernandez-Rapp J, Rainone S, Goupil C, Dorval V, Smith PY, Saint-Pierre M, 491 Vallee M, Planel E, Droit A, Calon Fet al (2016) microRNA-132/212 deficiency 492 enhances Abeta production and senile plaque deposition in Alzheimer's disease 493 triple transgenic mice. Scientific reports 6: 30953 Doi 10.1038/srep30953

49415 Hernandez-Rapp J, Smith PY, Filali M, Goupil C, Planel E, Magill ST, Goodman 495 RH, Hebert SS (2015) Memory formation and retention are affected in adult miR496 132/212 knockout mice. Behavioural brain research 287: 15-26 Doi 10.1016/j.bbr.2015.03.032

49816 Hoss AG, Kartha VK, Dong X, Latourelle JC, Dumitriu A, Hadzi TC, Macdonald 499 ME, Gusella JF, Akbarian S, Chen JFet al (2014) MicroRNAs located in the Hox 500 gene clusters are implicated in huntington's disease pathogenesis. PLoS Genet 10: $501 \quad$ e1004188 Doi 10.1371/journal.pgen.1004188

50217 Hoss AG, Labadorf A, Latourelle JC, Kartha VK, Hadzi TC, Gusella JF, 503 MacDonald ME, Chen JF, Akbarian S, Weng Zet al (2015) miR-10b-5p expression 
504 in Huntington's disease brain relates to age of onset and the extent of striatal

505 involvement. BMC medical genomics 8: 10 Doi 10.1186/s12920-015-0083-3

50618 Jin J, Cheng Y, Zhang Y, Wood W, Peng Q, Hutchison E, Mattson MP, Becker

$507 \quad \mathrm{KG}$, Duan W (2012) Interrogation of brain miRNA and mRNA expression profiles

508 reveals a molecular regulatory network that is perturbed by mutant huntingtin.

509 Journal of neurochemistry 123: 477-490 Doi 10.1111/j.1471-4159.2012.07925.x

51019 Kawahara Y, Mieda-Sato A (2012) TDP-43 promotes microRNA biogenesis as a

511 component of the Drosha and Dicer complexes. Proceedings of the National

512 Academy of Sciences of the United States of America 109: 3347-3352 Doi

$513 \quad 10.1073 /$ pnas. 1112427109

51420 Krauss S, Evert BO (2019) The Role of MicroRNAs in Spinocerebellar Ataxia

515 Type 3. Journal of molecular biology 431: 1729-1742 Doi

$516 \quad$ 10.1016/j.jmb.2019.01.019

$51721 \quad$ Kumar A, Vaish M, Ratan RR (2014) Transcriptional dysregulation in Huntington's

518 disease: a failure of adaptive transcriptional homeostasis. Drug Discov Today 19:

519 956-962 Doi 10.1016/j.drudis.2014.03.016

52022 Langfelder P, Gao F, Wang N, Howland D, Kwak S, Vogt TF, Aaronson JS,

521 Rosinski J, Coppola G, Horvath Set al (2018) MicroRNA signatures of endogenous

522 Huntingtin CAG repeat expansion in mice. PloS one 13: e0190550 Doi

$523 \quad$ 10.1371/journal.pone.0190550

52423 Lee ST, Chu K, Im WS, Yoon HJ, Im JY, Park JE, Park KH, Jung KH, Lee SK, 525 Kim Met al (2011) Altered microRNA regulation in Huntington's disease models.

526 Experimental neurology 227: 172-179 Doi 10.1016/j.expneurol.2010.10.012 
52724 Marti E, Pantano L, Banez-Coronel M, Llorens F, Minones-Moyano E, Porta S, 528 Sumoy L, Ferrer I, Estivill X (2010) A myriad of miRNA variants in control and 529 Huntington's disease brain regions detected by massively parallel sequencing. $530 \quad$ Nucleic acids research 38: 7219-7235 Doi 10.1093/nar/gkq575

53125 Martinez B, Peplow PV (2021) Altered microRNA expression in animal models of 532 Huntington's disease and potential therapeutic strategies. Neural Regen Res 16: 533 2159-2169 Doi 10.4103/1673-5374.310673

53426 McCann C, Holohan EE, Das S, Dervan A, Larkin A, Lee JA, Rodrigues V, Parker 535 R, Ramaswami M (2011) The Ataxin-2 protein is required for microRNA function 536 and synapse-specific long-term olfactory habituation. Proceedings of the National 537 Academy of Sciences of the United States of America 108: E655-662 Doi $538 \quad 10.1073 /$ pnas. 1107198108

53927 O'Brien J, Hayder H, Zayed Y, Peng C (2018) Overview of MicroRNA Biogenesis, 540 Mechanisms of Actions, and Circulation. Front Endocrinol (Lausanne) 9: 402 Doi $541 \quad 10.3389 /$ fendo.2018.00402

54228 Olmo IG, Olmo RP, Goncalves ANA, Pires RGW, Marques JT, Ribeiro FM (2021) 543 High-Throughput Sequencing of BACHD Mice Reveals Upregulation of $544 \quad$ Neuroprotective miRNAs at the Pre-Symptomatic Stage of Huntington's Disease. $545 \quad$ ASN Neuro 13: 17590914211009857 Doi 10.1177/17590914211009857

54629 Pircs K, Petri R, Madsen S, Brattas PL, Vuono R, Ottosson DR, St-Amour I, 547 Hersbach BA, Matusiak-Bruckner M, Lundh SHet al (2018) Huntingtin 548 Aggregation Impairs Autophagy, Leading to Argonaute-2 Accumulation and 
55130 Pogoda A, Chmielewska N, Maciejak P, Szyndler J (2021) Transcriptional

552 Dysregulation in Huntington's Disease: The Role in Pathogenesis and Potency for 553 Pharmacological Targeting. Current medicinal chemistry 28: 2783-2806 Doi $554 \quad 10.2174 / 0929867327666200705225821$

55531 Ratovitski T, Gucek M, Jiang H, Chighladze E, Waldron E, D'Ambola J, Hou Z, 556 Liang Y, Poirier MA, Hirschhorn RRet al (2009) Mutant huntingtin N-terminal 557 fragments of specific size mediate aggregation and toxicity in neuronal cells. The $558 \quad$ Journal of biological chemistry 284: 10855-10867 Doi 10.1074/jbc.M804813200

55932 Rieux M, Alpaugh M, Sciacca G, Saint-Pierre M, Masnata M, Denis HL, Levesque 560 SA, Herrmann F, Bazenet C, Garneau APet al (2020) Shedding a new light on 561 Huntington's disease: how blood can both propagate and ameliorate disease 562 pathology. Molecular psychiatry: Doi 10.1038/s41380-020-0787-4

56333 Ross CA, Tabrizi SJ (2011) Huntington's disease: from molecular pathogenesis to 564 clinical treatment. The Lancet Neurology 10: 83-98 Doi 10.1016/S1474$565 \quad 4422(10) 70245-3$

56634 Samadi P, Boutet A, Rymar VV, Rawal K, Maheux J, Kvann JC, Tomaszewski M, 567 Beaubien F, Cloutier JF, Levesque Det al (2013) Relationship between BDNF 568 expression in major striatal afferents, striatum morphology and motor behavior in 569 the R6/2 mouse model of Huntington's disease. Genes Brain Behav 12: 108-124 Doi 10.1111/j.1601-183X.2012.00858.X 
57135 Savas JN, Ma B, Deinhardt K, Culver BP, Restituito S, Wu L, Belasco JG, Chao 572 MV, Tanese N (2010) A role for huntington disease protein in dendritic RNA 573 granules. The Journal of biological chemistry 285: 13142-13153 Doi $574 \quad 10.1074 / j b c . M 110.114561$

57536 Savas JN, Makusky A, Ottosen S, Baillat D, Then F, Krainc D, Shiekhattar R, 576 Markey SP, Tanese N (2008) Huntington's disease protein contributes to RNA577 mediated gene silencing through association with Argonaute and P bodies. 578 Proceedings of the National Academy of Sciences of the United States of America 579 105: 10820-10825 Doi 10.1073/pnas.0800658105

58037 Seitz H (2019) On the Number of Functional microRNA Targets. Mol Biol Evol $581 \quad 36: 1596-1597$ Doi 10.1093/molbev/msz054

58238 Sellier C, Freyermuth F, Tabet R, Tran T, He F, Ruffenach F, Alunni V, Moine H, 583 Thibault C, Page Aet al (2013) Sequestration of DROSHA and DGCR8 by 584 expanded CGG RNA repeats alters microRNA processing in fragile X-associated 585 tremor/ataxia syndrome. Cell reports 3: 869-880 Doi 10.1016/j.celrep.2013.02.004 58639 Smith PY, Delay C, Girard J, Papon MA, Planel E, Sergeant N, Buee L, Hebert SS (2011) MicroRNA-132 loss is associated with tau exon 10 inclusion in progressive 588 supranuclear palsy. Human molecular genetics 20: 4016-4024 Doi $589 \quad 10.1093 / \mathrm{hmg} / \mathrm{ddr} 330$

59040 St-Amour I, Turgeon A, Goupil C, Planel E, Hebert SS (2018) Co-occurrence of 591 mixed proteinopathies in late-stage Huntington's disease. Acta neuropathologica 592 135: 249-265 Doi 10.1007/s00401-017-1786-7 
59341 Vidigal JA, Ventura A (2015) The biological functions of miRNAs: lessons from 594 in vivo studies. Trends in cell biology 25: 137-147 Doi 10.1016/j.tcb.2014.11.004

59542 Wilczynska A, Bushell M (2015) The complexity of miRNA-mediated repression.

$596 \quad$ Cell death and differentiation 22: 22-33 Doi 10.1038/cdd.2014.112

59743 Wu Y, Dai X, Ni Z, Yan X, He F, Lian J (2017) The downregulation of ATG4B 598 mediated by microRNA-34a/34c-5p suppresses rapamycin-induced autophagy. $599 \quad$ Iran J Basic Med Sci 20: 1125-1130 Doi 10.22038/IJBMS.2017.9446

60044 Zhou H, Cao F, Wang Z, Yu ZX, Nguyen HP, Evans J, Li SH, Li XJ (2003) 601 Huntingtin forms toxic NH2-terminal fragment complexes that are promoted by the 602 age-dependent decrease in proteasome activity. The Journal of cell biology 163: $603 \quad 109-118$ Doi $10.1083 /$ jcb.200306038

60445 Zuccato C, Cattaneo E (2007) Role of brain-derived neurotrophic factor in 605 Huntington's disease. Prog Neurobiol 81: 294-330 Doi $606 \quad$ 10.1016/j.pneurobio.2007.01.003

610 Figure 1. Differential Htt pathology between HD striatum and cortex. Representative 611 immunoblots of endogenous full-length Huntingtin (Htt) (1HU-4C8 antibody) and N612 terminal Htt fragments (1HU-4C8 antibody) in the soluble fraction of (A) the striatum or 613 (B) the cortex of HD patients and Controls. (C-E) Protein quantifications of soluble full614 length Htt (1HU-4C8 and CH00146 antibodies) and N-terminal Htt (1HU-4C8 antibody). 615 Representative dot blots of formic acid (FA)-insoluble full-length Htt (1HU-4C8) and 
616 mutant Htt (Poly-Gln) in (F) the striatum and $(\mathbf{G})$ the cortex of HD patients and Controls.

617 (H-I) Protein quantifications of dot blots. Representative immunoblots of endogenous

618 NeuN, Darpp32 and PSD95 in $(\mathbf{J})$ the striatum or $(\mathbf{K})$ the cortex on HD patients and

619 Controls with quantifications in (L-M). Bar graphs with standard error of the mean (SEM)

620 are shown, where the average of Controls is set as $100 \%$. In all cases, the HD group is

621 presented as pooled or per stage. Statistics: Ctl vs. HD as a group was calculated using a

622 Mann-Whitney test. Ctl vs. HD stages was calculated using an analysis of covariance

623 followed by Kruskal-Wallis multiple comparison test. Significant fold changes are

624 provided for each group. * $P<0.05$; ** $P<0.01$; *** $P<0.001$; **** $P<0.0001$.

625 Abbreviations: Ctl, Controls; HD, Huntington's disease; HD2, Vonsattel grade 2; HD3,

626 Vonsattel grade 3; HD4, Vonsattel grade 4.

628 Figure 2. miRNA biogenesis components go awry in human HD striatum.

629 Representative immunoblots of endogenous DGCR8, TRBP, Drosha, Dicer, Ago2 and

630 Exportin in the soluble fraction of (A) the striatum or (B) the cortex of HD patients and

631 Controls. See Methods for the list of antibodies. (C-E) Protein quantifications of each

632 protein according to disease or brain region. Bar graphs with standard error of the mean

633 (SEM) are shown, where the average of Controls is set as 100\%. In all cases, the HD group

634 is presented as pooled or per stage. Statistics: Ctl vs. HD as a group was calculated using 635 a Mann-Whitney test. Ctl vs. HD stages was calculated using an analysis of covariance 636 followed by Kruskal-Wallis comparison test. Significant fold changes are provided for 637 each group. * $P<0.05$; ** $P<0.01$; *** $P<0.001$; **** $P<0.0001$. Abbreviations: Ctl, 
638 Controls; HD, Huntington's disease; HD2, Vonsattel grade 2; HD3, Vonsattel grade 3;

639 HD4, Vonsattel grade 4.

640

641 Figure 3. Imbalance between miRNA maturation products in HD brain. (A-F) Side-

642 by-side comparison of selected primary, precursor, and mature miRNA transcripts in the

643 striatum of HD patients and Controls. In all assays, we used probe-specific miRNA

644 quantitative RT-PCRs. See Methods for normalization procedures. (G-L). A similar

645 analysis of the same miRNAs in matching cortex samples. Bar graphs with standard error

646 of the mean (SEM) are shown, where the average of Controls is set as $100 \%$ for each

647 miRNA species. Statistics: Ctl vs. HD as a group was calculated using a Mann-Whitney

648 test. Ctl vs. HD stages was calculated using an analysis of covariance followed by Kruskal-

649 Wallis multiple comparison test. Significant fold changes are provided for each group. *

$650 P<0.05$; ** $P<0.01$; *** $P<0.001$; **** $P<0.0001$. Trends are shown as well.

651 Abbreviations: Ctl, Controls; HD, Huntington's disease; HD2, Vonsattel grade 2; HD3,

652 Vonsattel grade 3; HD4, Vonsattel grade 4.

653

654 Figure 4. Predominant pre- to mature miRNA maturation deficits in HD brain. (A-

655 F) Overview of candidate miRNA primary/precursor inhibition scores (ratios) in the

656 striatum and cortex of HD patients and Controls. Graphs were generated using

657 corresponding qRT-PCR data. Significant differences were observed for a subset of tested

658 miRNAs. (G-L) Overview of miRNA precursor/mature inhibition scores (ratios) in the

659 striatum and cortex of HD patients and Controls. Here, all the tested miRNAs were

660 significantly affected, particularly in the striatum. Statistics: Ctl vs. HD as a group was 
661 calculated using a Mann-Whitney test. Ctl vs. HD stages was calculated using an analysis

662 of covariance followed by Kruskal-Wallis multiple comparison test. Significant fold

663 changes are provided for each group. * $P<0.05$; ** $P<0.01 ; * * * P<0.001 ; * * * * P<0.0001$.

664 Trends are shown as well. Abbreviations: Ctl, Controls; HD, Huntington's disease; HD2,

665 Vonsattel grade 2; HD3, Vonsattel grade 3; HD4, Vonsattel grade 4.

666

667 Figure 5. Altered autophagy in HD brain. Representative immunoblots of endogenous

668 ATG9a, P62, LC3, Beclin, ATG5a, and TDP-43 in the soluble fraction of (A) the striatum

669 or (B) the cortex of HD patients and Controls. See Methods for the list of antibodies. (C-

670 E) Protein quantifications of each protein according to disease or brain region. Bar graphs

671 with standard error of the mean (SEM) are shown, where the average of Controls is set as

$672100 \%$. In all cases, the HD group is presented as pooled or per stage. Statistics: Ctl vs. HD

673 as a group was calculated using a Mann-Whitney test. Ctl vs. HD stages was calculated

674 using an analysis of covariance followed by Kruskal-Wallis multiple comparison test.

675 Significant fold changes are provided for each group. ${ }^{*} P<0.05 ; * * P<0.01$; *** $P<0.001$;

$676 * * * * P<0.0001$. Abbreviations: Ctl, Controls; HD, Huntington's disease; HD2, Vonsattel

677 grade 2; HD3, Vonsattel grade 3; HD4, Vonsattel grade 4. 
bioRxiv preprint doi: https://doi.org/10.1101/2022.01.27.478047; this version posted January 28, 2022. The copyright holder for this preprint (which was not certified by peer review) is the author/funder. All rights reserved. No reuse allowed without permission.
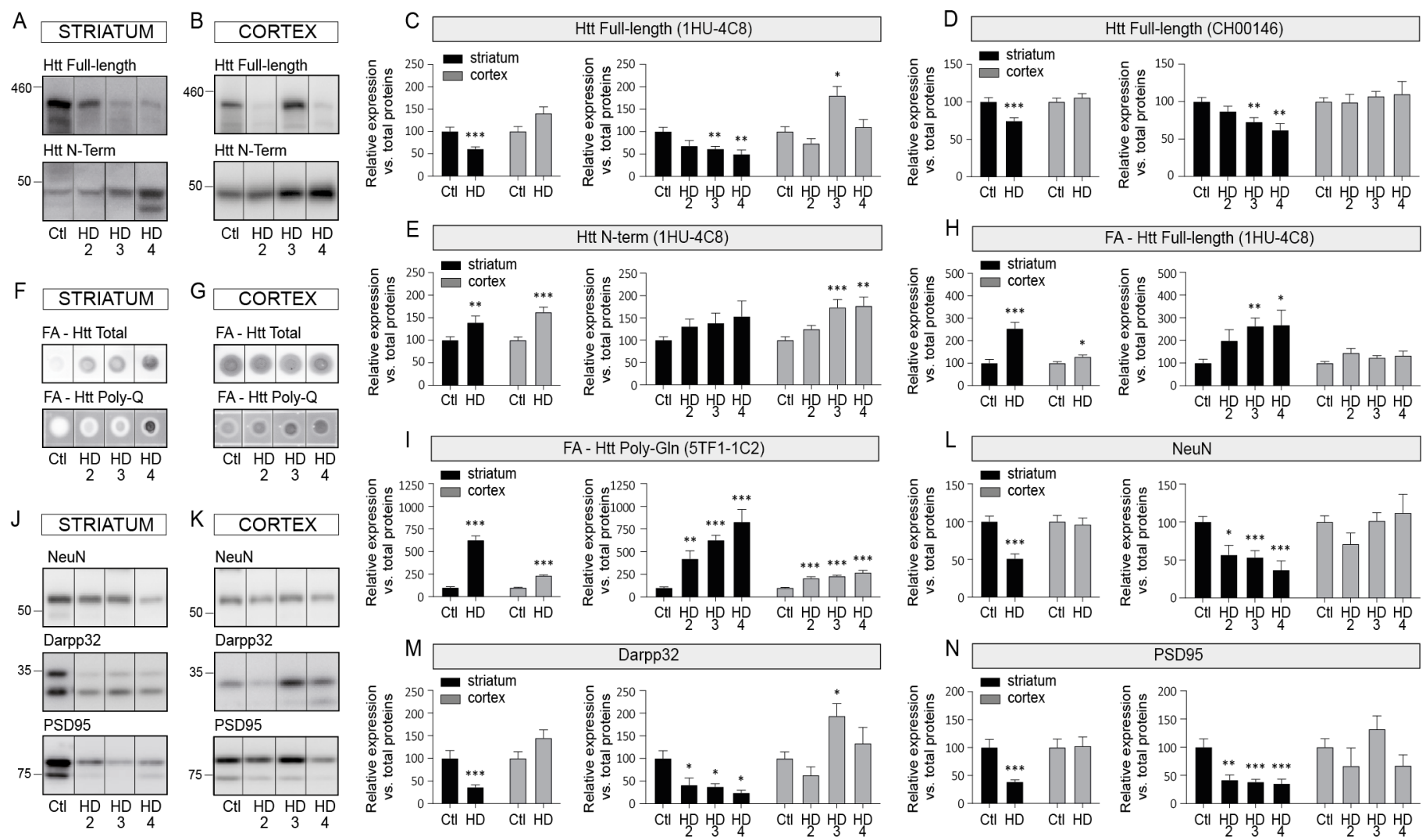

FA - Htt Poly-GIn (5TF1-1C

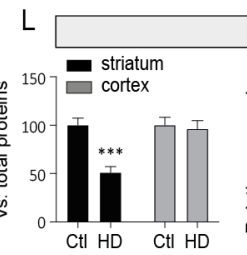

NeuN
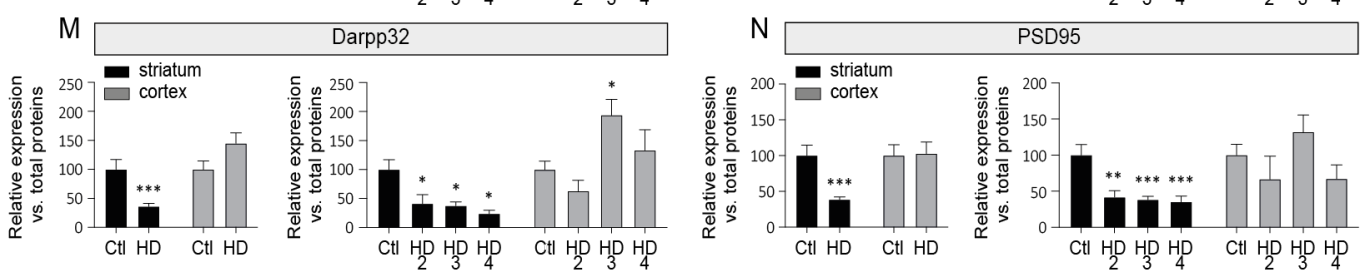

FIGURE 1 
bioRxiv preprint doi: https://doi.org/10.1101/2022.01.27.478047; this version posted January 28, 2022. The copyright holder for this preprint (which was not certified by peer review) is the author/funder. All rights reserved. No reuse allowed without permission.
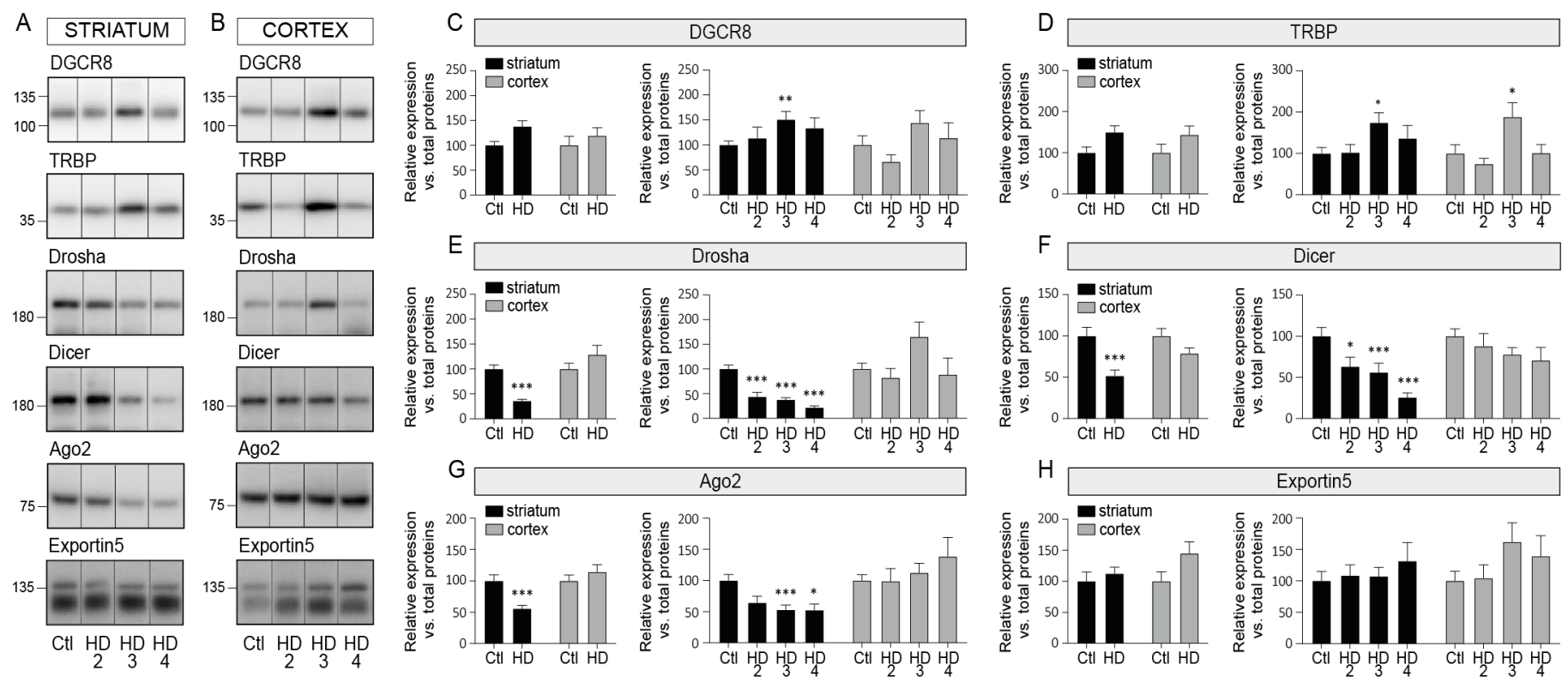

FIGURE 2 


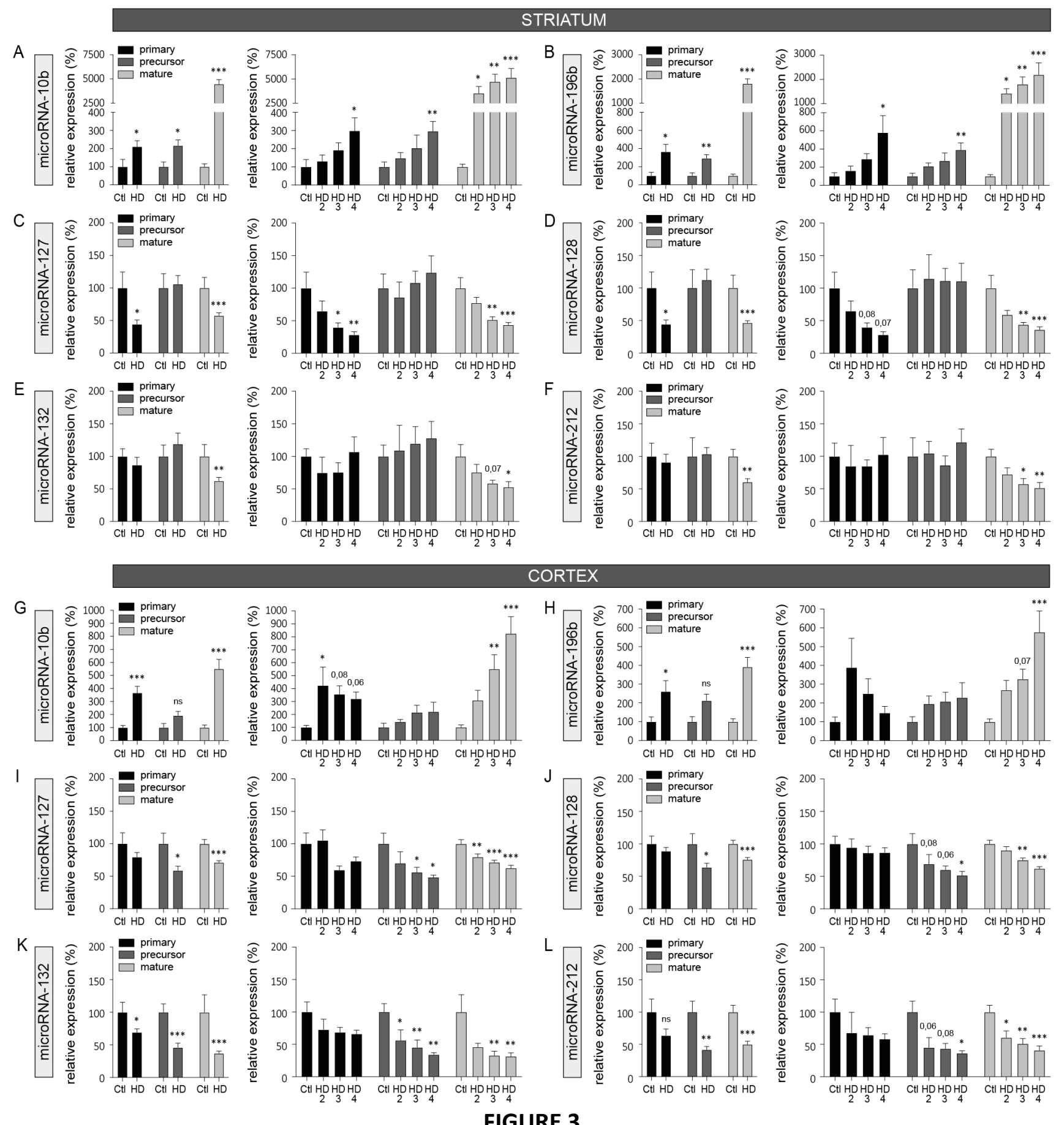


bioRxiv preprint doi: https://doi.org/10.1101/2022.01.27.478047; this version posted January 28, 2022. The copyright holder for this preprint (which was not certified by peer review) is the author/funder. All rights reserved. No reuse allowed without permission.

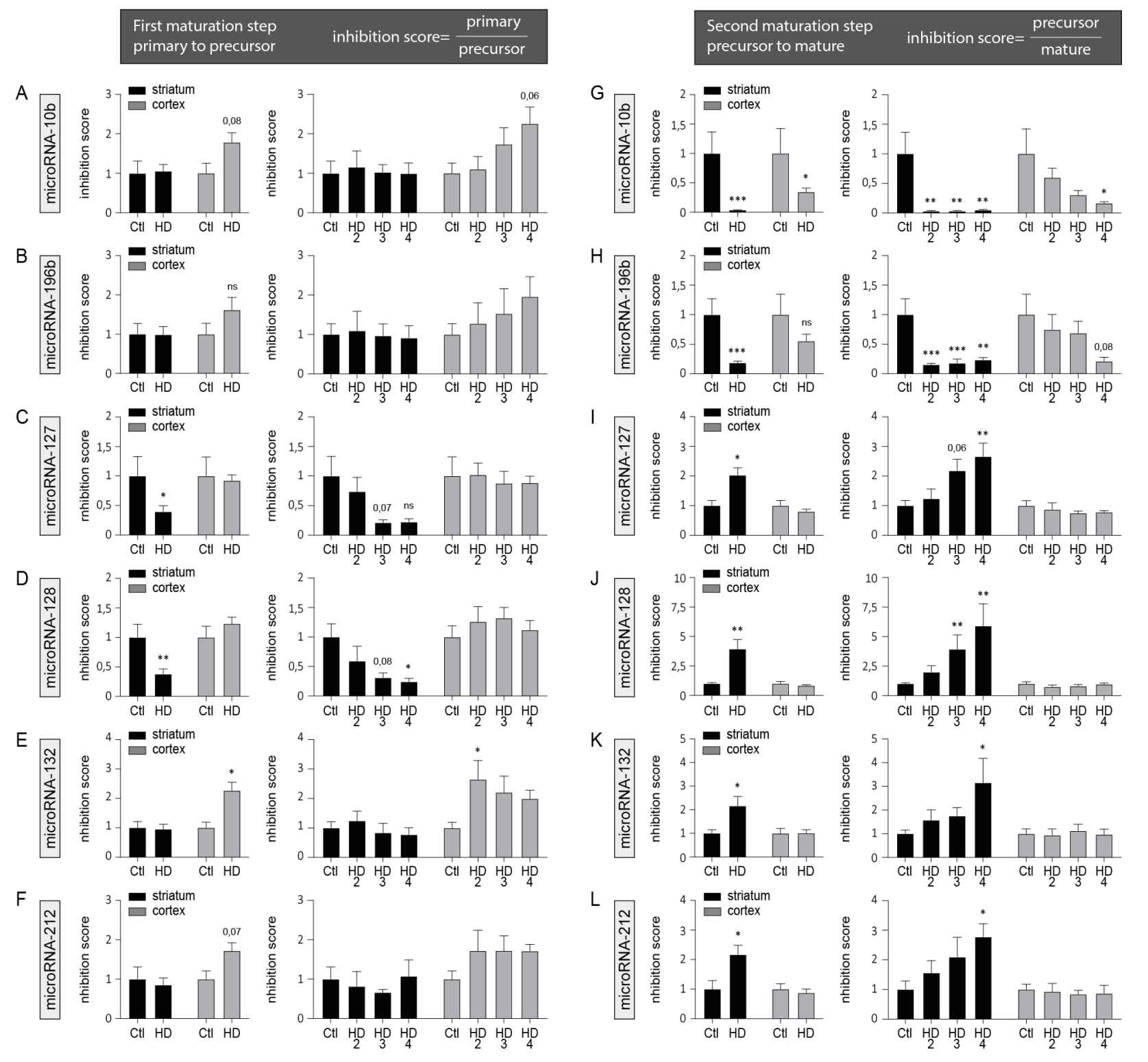

FIGURE 4 
bioRxiv preprint doi: https://doi.org/10.1101/2022.01.27.478047; this version posted January 28, 2022. The copyright holder for this preprint (which was not certified by peer review) is the author/funder. All rights reserved. No reuse allowed without permission.

A STRIATUM ATG9a

135

$100--=$

P62

$35-$

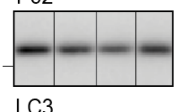

LC3

$180-$

Beclin

$180-$

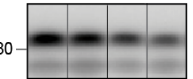

ATG5a

$75-\square=-$

TDP43

$135-$

CHD

Ctl $\underset{2}{\mathrm{HD}} \underset{3}{\mathrm{HD}} \mathrm{HD}$
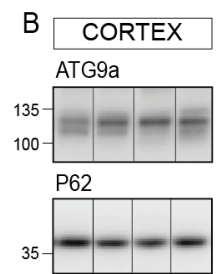

LC3

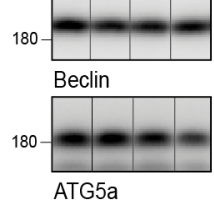

ATG5a

$75-=2$

TDP43

$135-$\begin{tabular}{|l|l|}
\hline & \\
- &
\end{tabular}

Ctl $\underset{2}{\mathrm{HD}} \underset{3}{\mathrm{HD}} \mathrm{HD}$

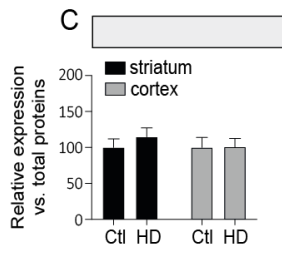

ATG9a
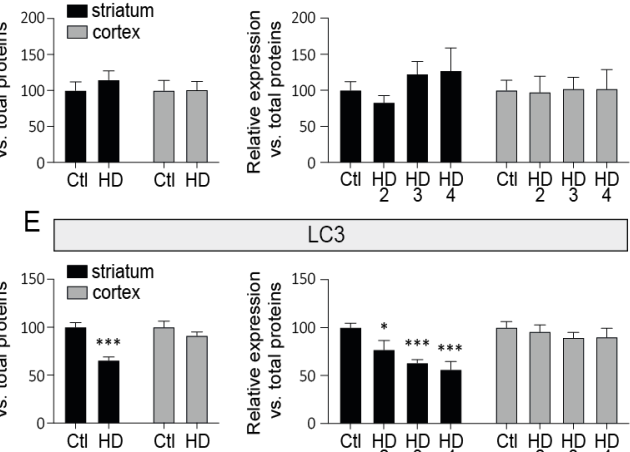

LC3

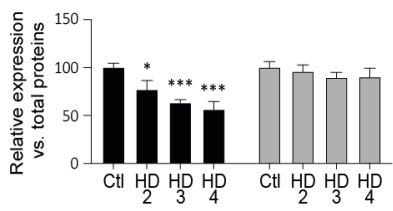

\section{G}

ATG5a
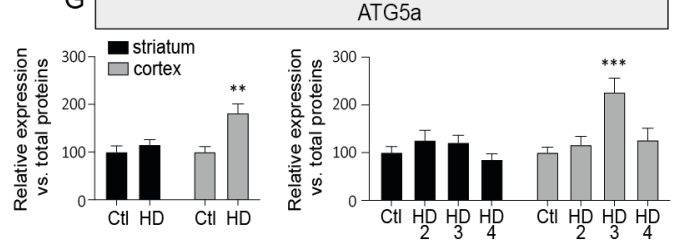
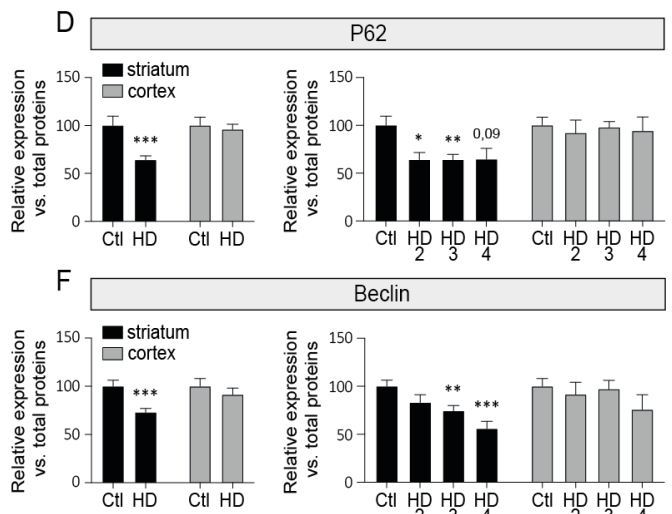

Beclin
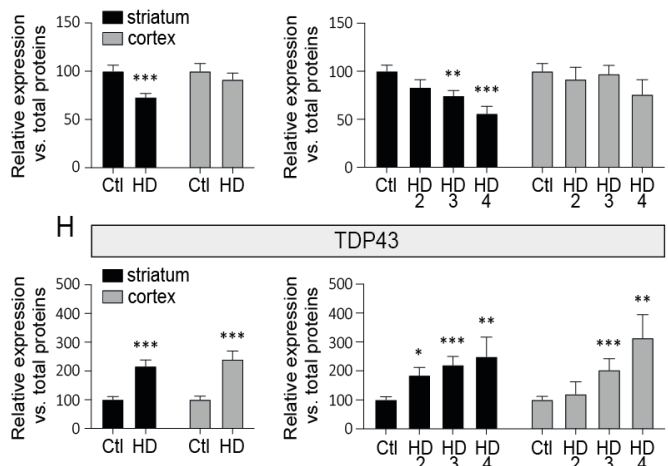

TDP43

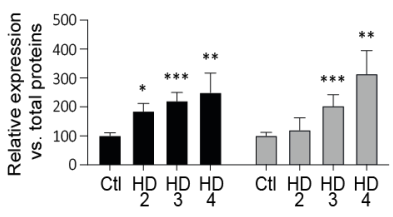

FIGURE 5 